\title{
Distributed-Flap Layout Trade Study on a Highly Flexible Common Research Model
}

David L Rodriguez Michael Aftosmis Marian Nemec George Anderson

\author{
June 27, 2018 \\ Aviation Forum
}

NASA Ames Research Center 


\section{VCCTEF Concept}

- Variable Camber Continuous Trailing Edge Flaps

- Flaps distributed over most of the span of the wing

- Elastomer material between flaps to seal gaps

- Tailors spanwise lift distribution throughout mission

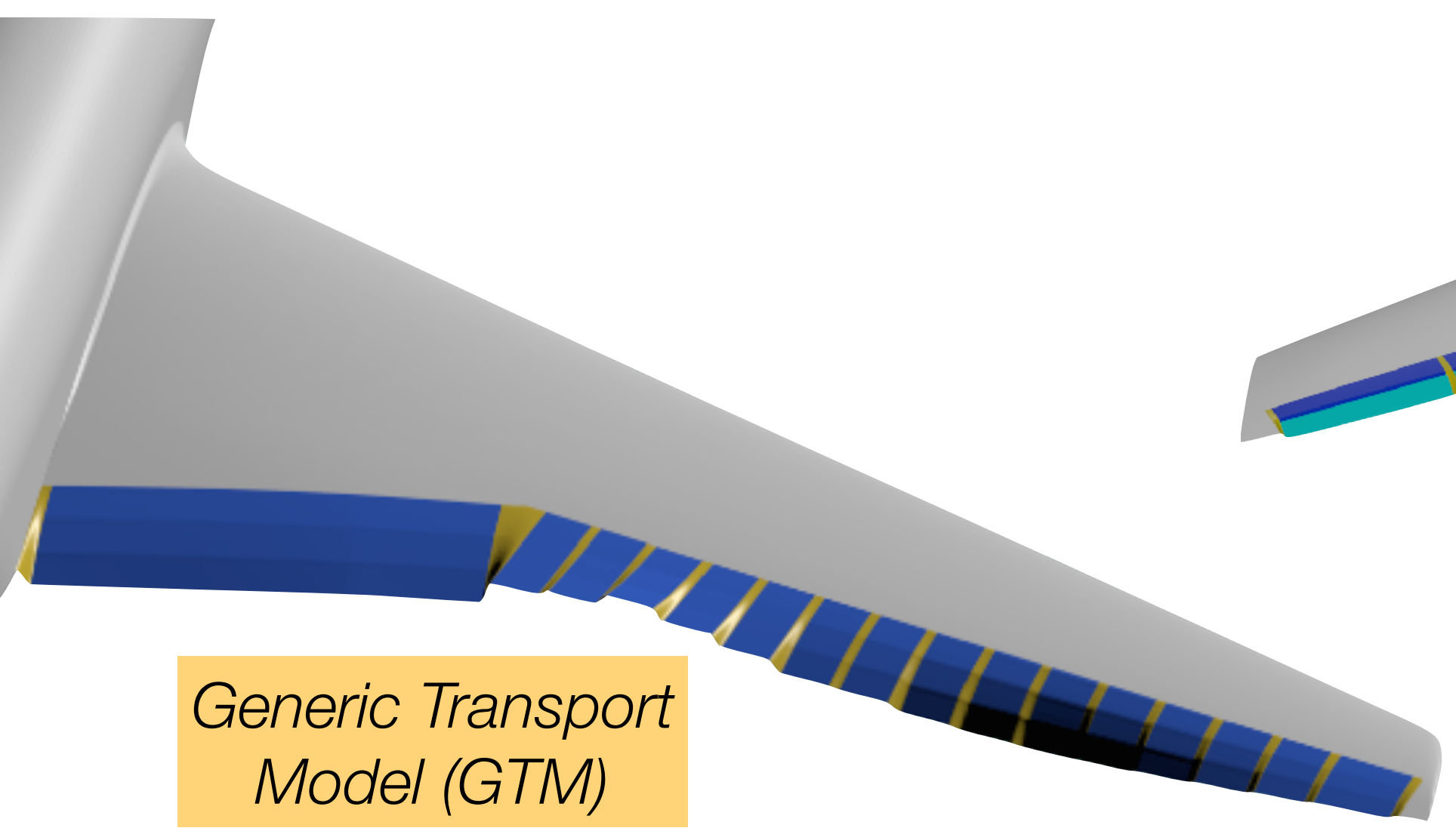




\section{Motivation}

- Early application of VCCTEF on GTM on overspeed case indicated wave drag could also be significantly reduced

- More effective if circular deflection relaxed
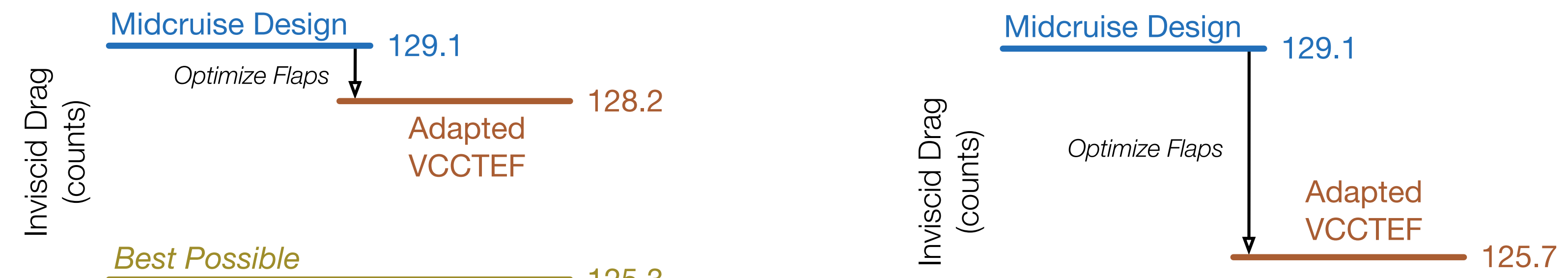

Best Possible

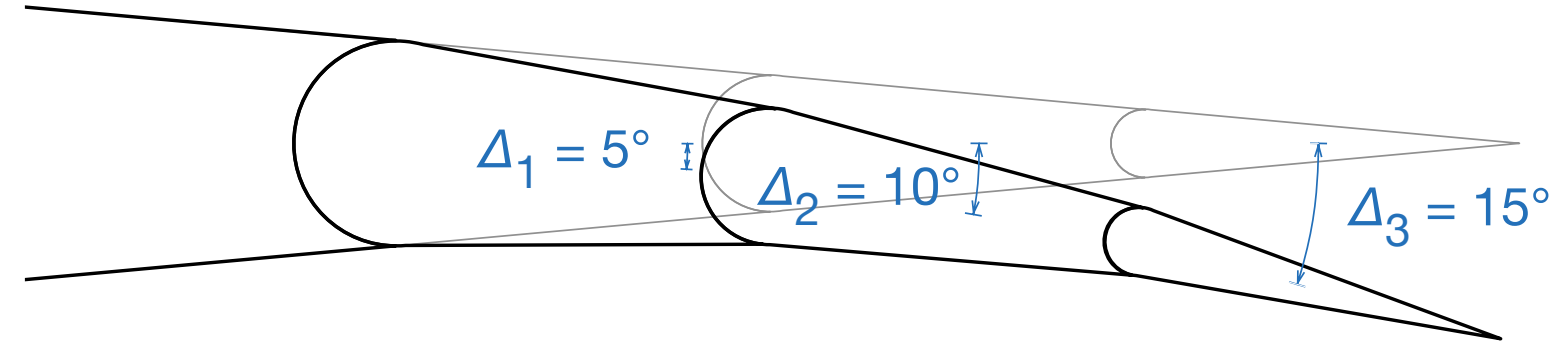

$$
\Delta_{2}=2 \Delta_{1}, \Delta_{3}=3 \Delta_{1}
$$




\section{Goals and Methods}

- Determine how complex a distributed flap system must be to be effective for overspeed drag reduction

- how many spanwise flaps?

- how many chordwise segments per flap?

- Flap layout trade study

- Install various layouts with different number of spanwise flaps and chordwise segments

- Optimize flap deflections on all layouts at overspeed condition

- Examine results for trends

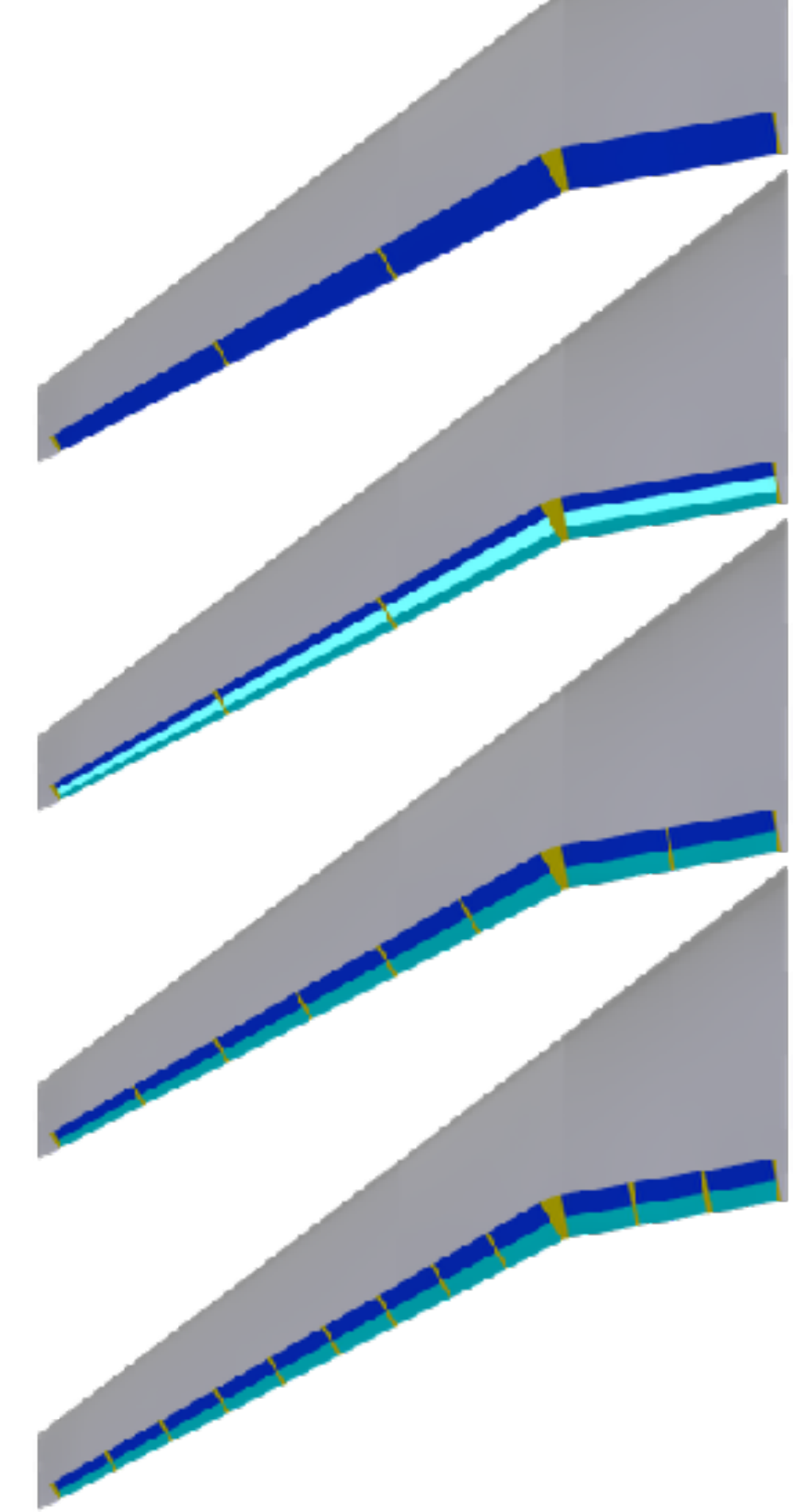




\section{Platform for Trade Study}

- Common Research Model (CRM) fuselage/wing/horizontal tail configuration)

- Assume composite wing

- remove built in deformation from original CRM geometry

- develop structural model that exhibits greater deformation (about twice original)

- Develop new baseline wing

- start with original CRM geometry

- re-optimize twist distribution for cruise using methods that address aeroelastic effects

- minimize drag

- constrain lift

- maintain trim

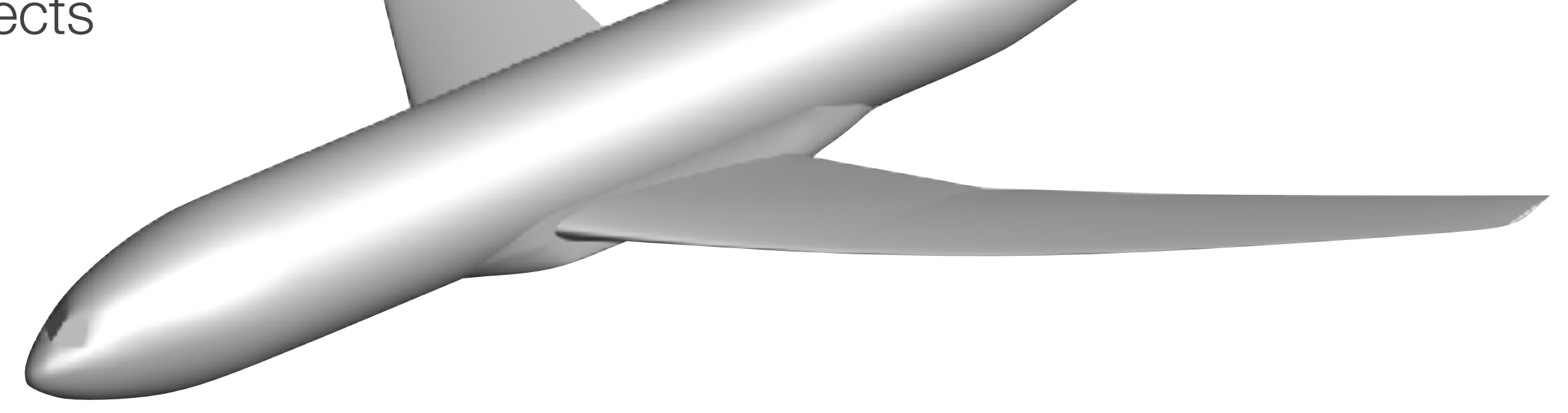




\section{Static Aeroelastic Analysis Architecture}

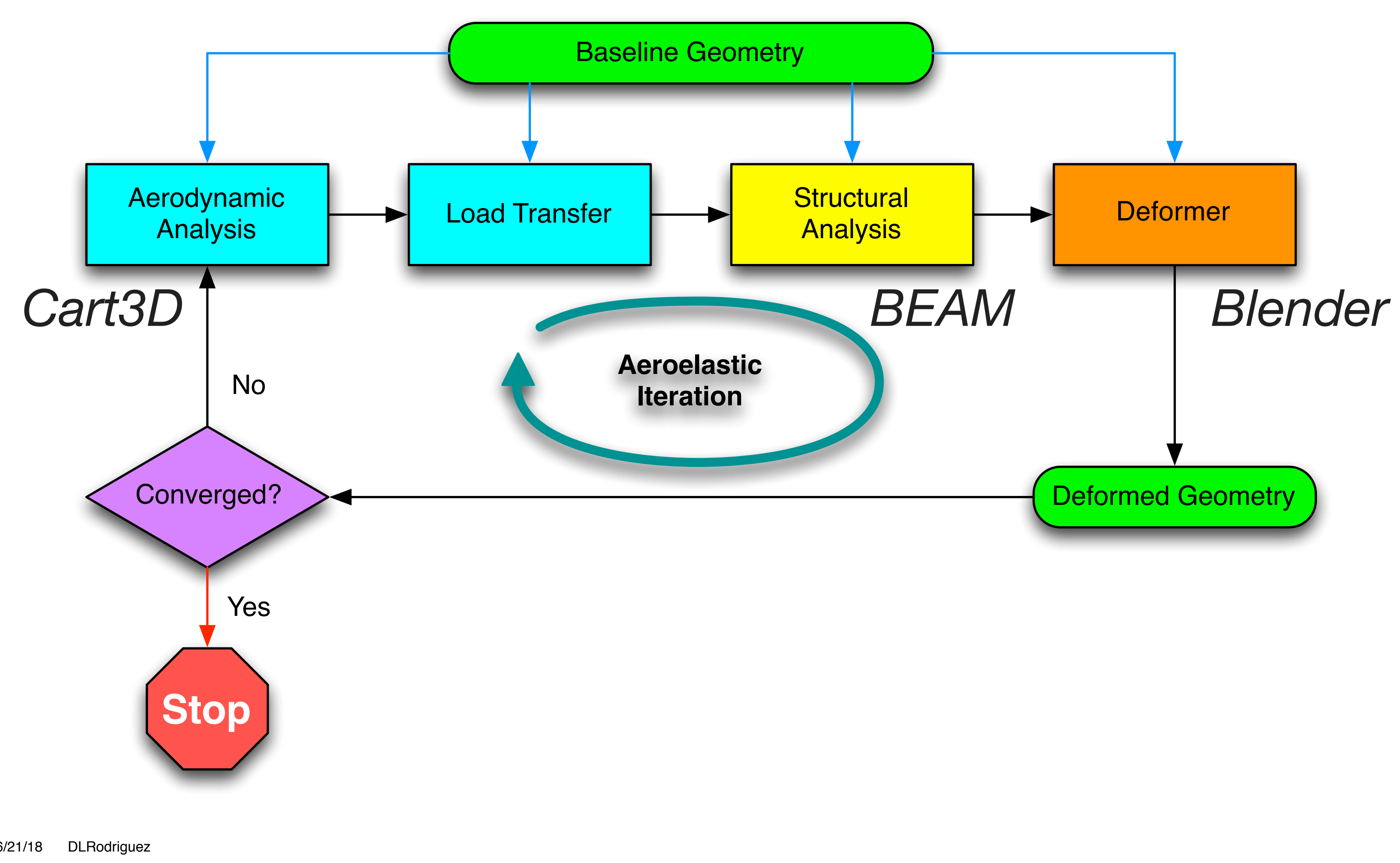




\section{Aerodynamic Shape Optimization Architecture}

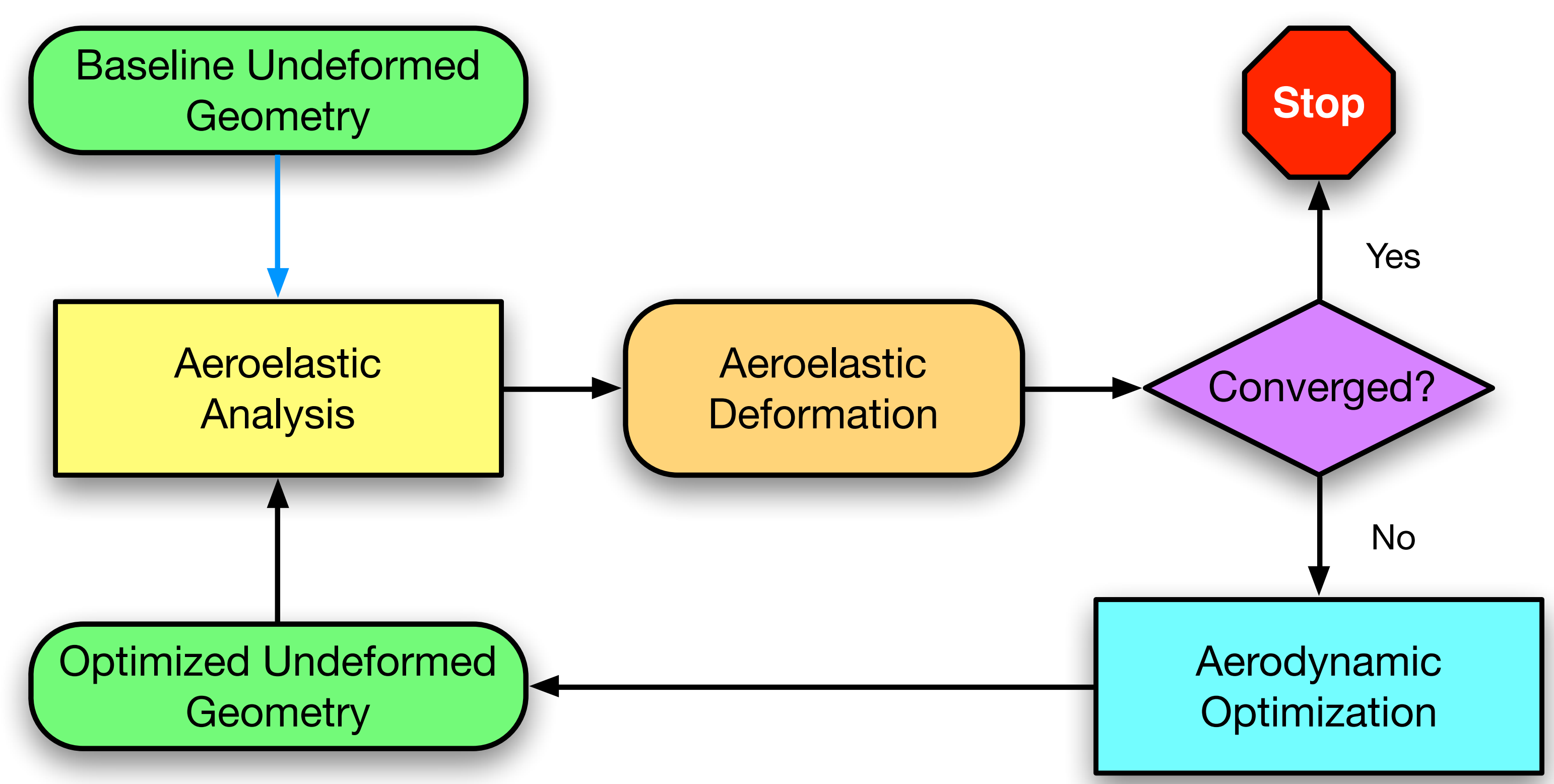




\section{Modeling the VCCTEF}

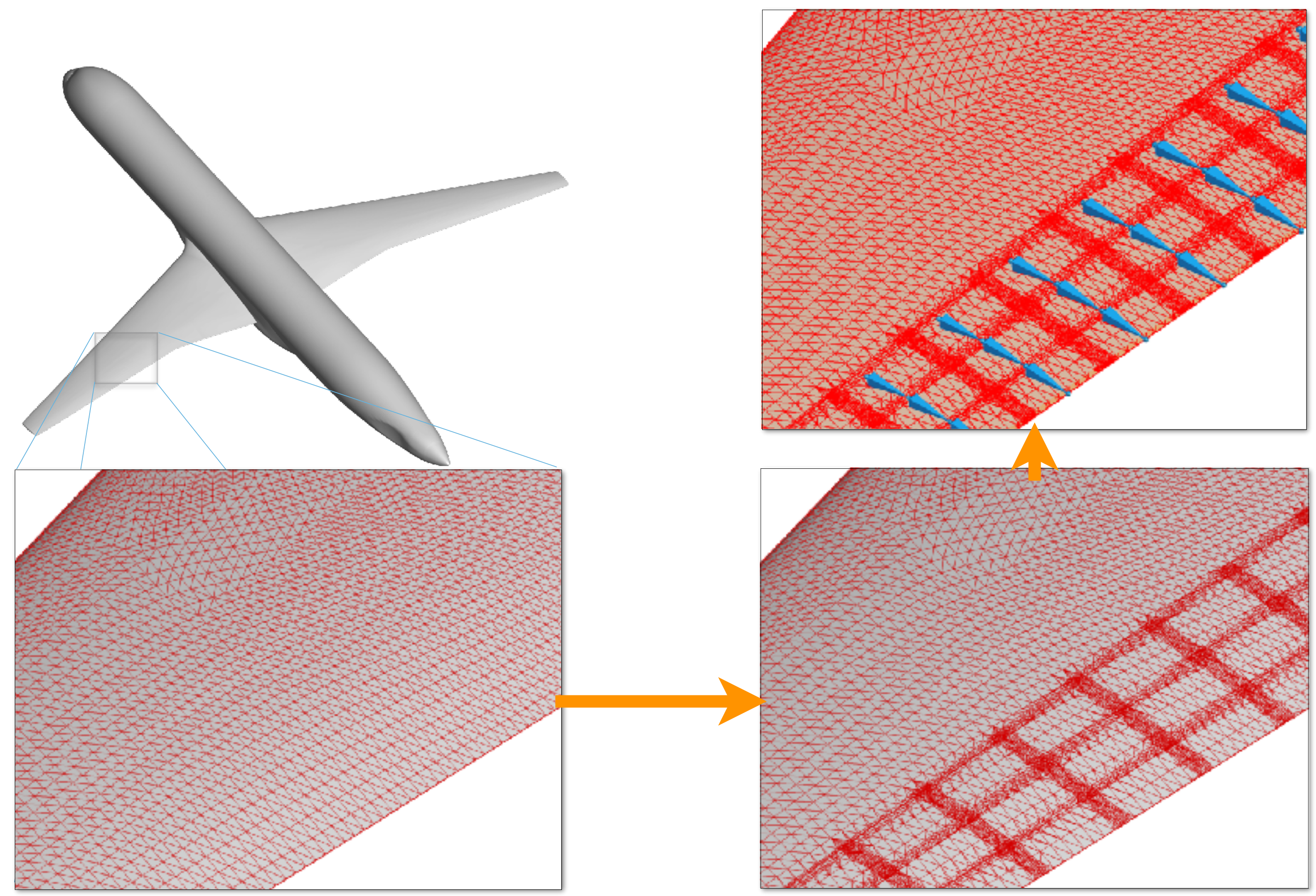




\section{Modeling the VCCTEF}

- Flap deflections controlled by Blender "armature" (analogous to a skeleton)

- Surface triangulation is bound to "bones"

- Bones can only rotate about hinge lines

- Sequential flaps bones linked to each other

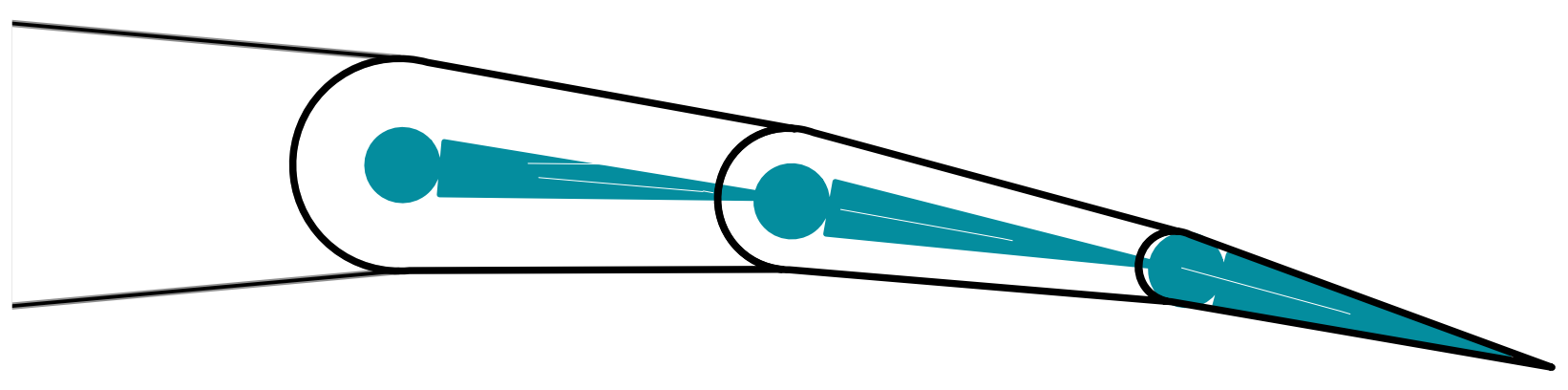

- Blended transition between flaps to mimic elastomer material
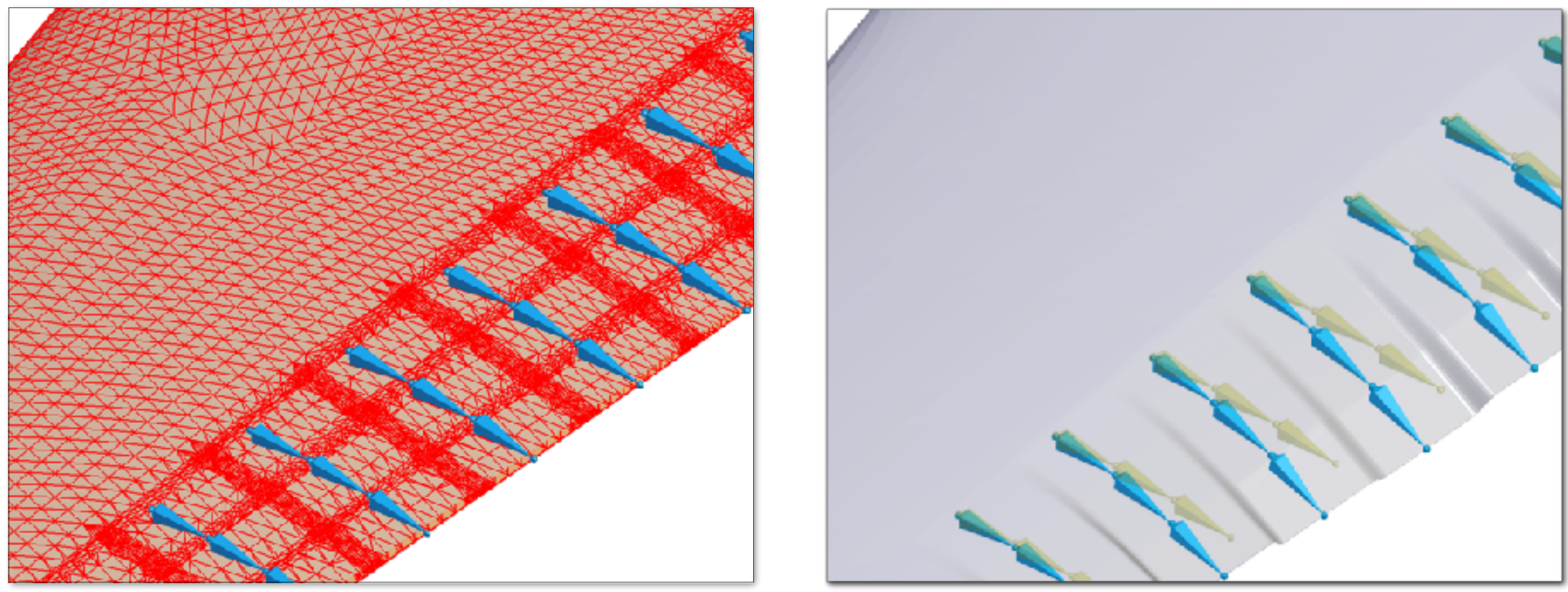


\section{Establishing a New Baseline CRM Design}

- CRM wing twist distribution reoptimized with more flexible structure

- Minimize drag $\left(C_{D}\right)$ at cruise condition $\left(M_{\infty}=0.85\right)$

- maintain cruise lift $\left(C_{L}=0.5\right)$

- maintain longitudinal trim $\left(C_{M}=0\right)$

- cabin deck angle constraint $\left(\boldsymbol{\alpha}_{\max }=3^{\circ}\right)$

- Design variables

- section incidence at 6 spanwise stations (including root), while linearly vary change in incidence between stations

- angle of attack (helps satisfy lift constraint)

- tail incidence (helps satisfy trim) minimize: $\quad C_{D}\left(\alpha, \boldsymbol{\theta}_{w}, i_{t}\right)$

subject to: $\quad C_{L}\left(\alpha, \boldsymbol{\theta}_{w}, i_{t}\right)=C_{L, \text { cruise }}=0.5$

$C_{M}\left(\alpha, \boldsymbol{\theta}_{w}, i_{t}\right)=0$

$\alpha \leq \alpha_{\max }$

Outboard 2

\section{Outboard 1}
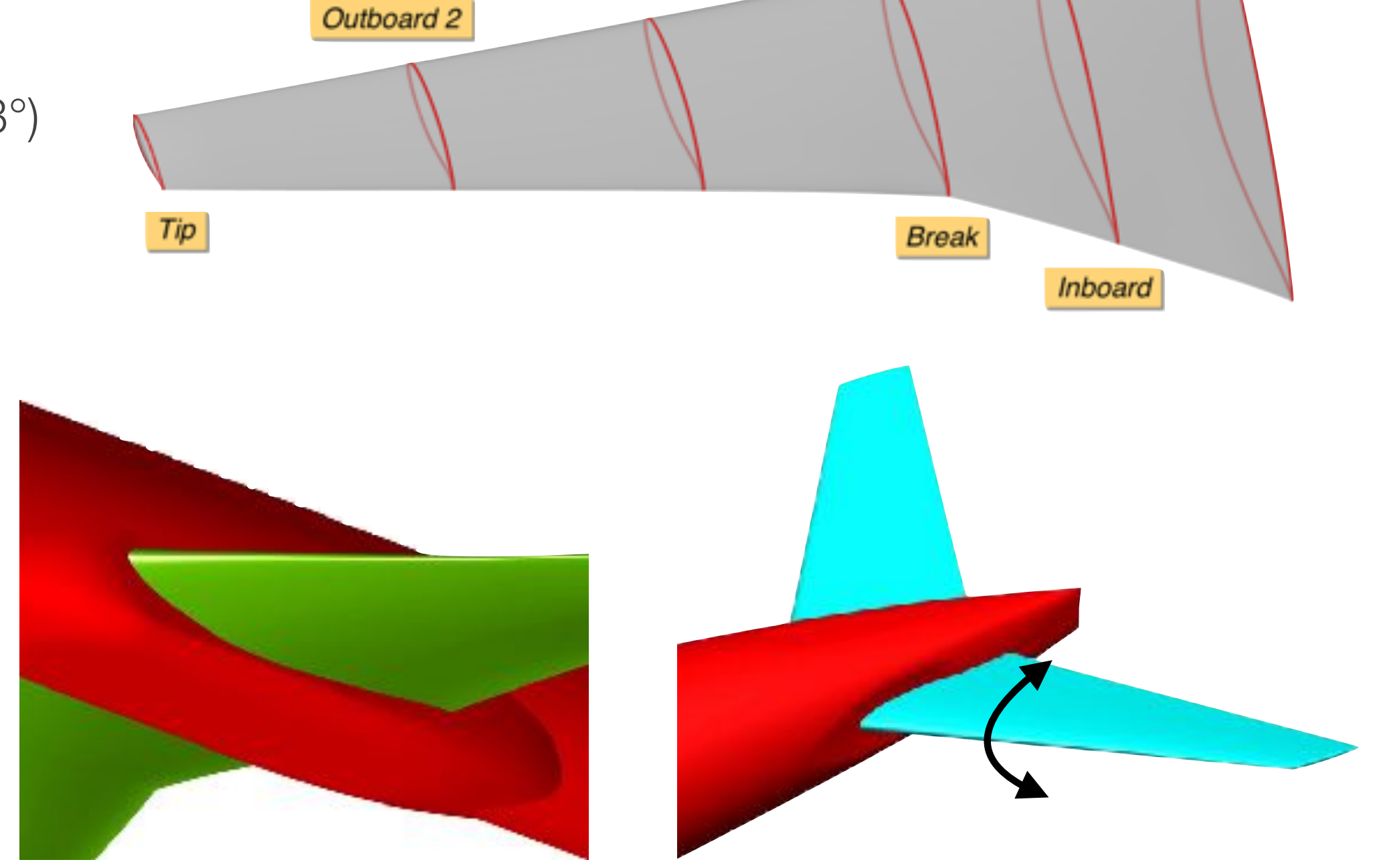


\section{Convergence of Twist Optimization}

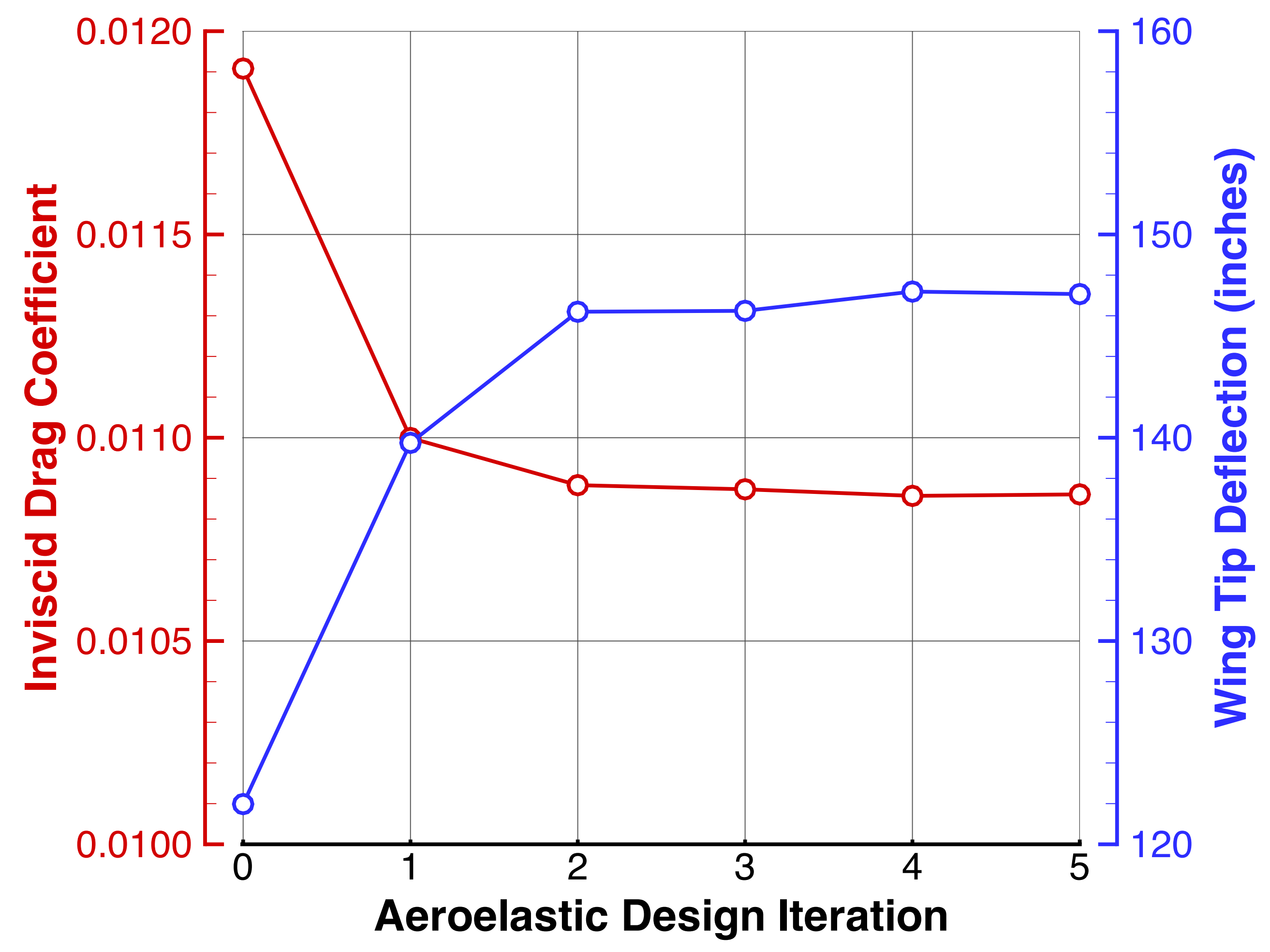




\section{Convergence of Twist Optimization}

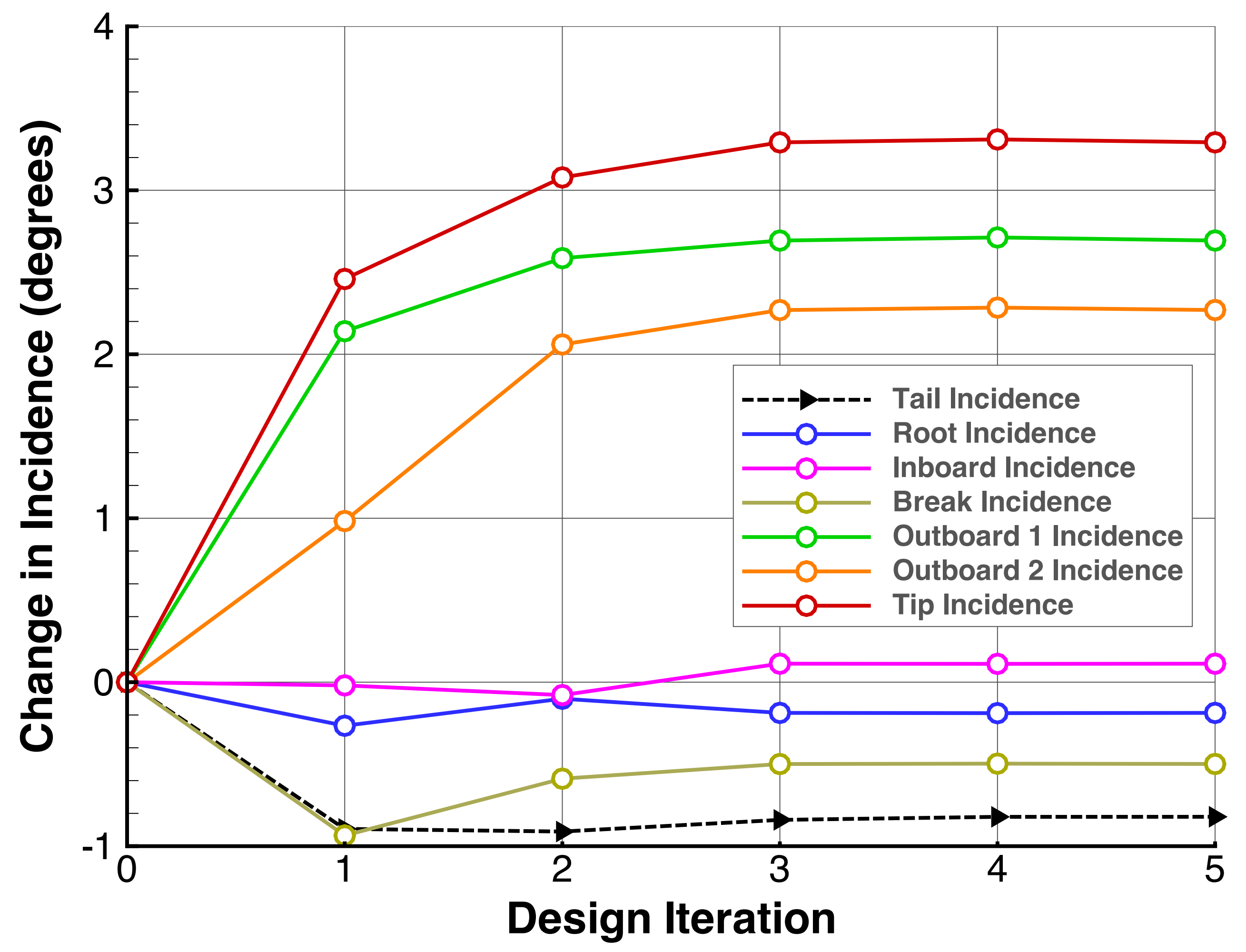




\section{Optimized Twist Distribution}

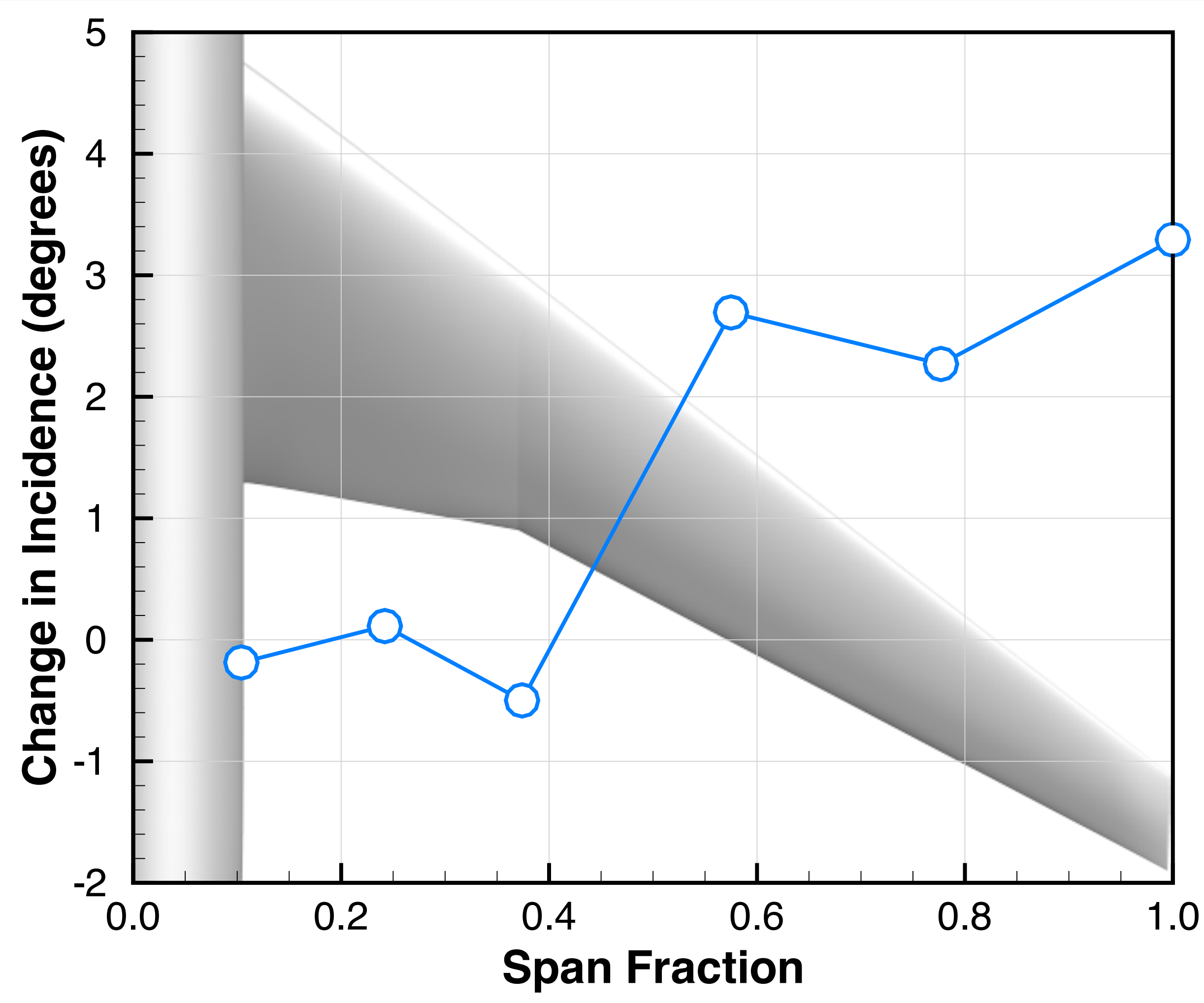




\section{Optimized Spanwise Lift Distribution}

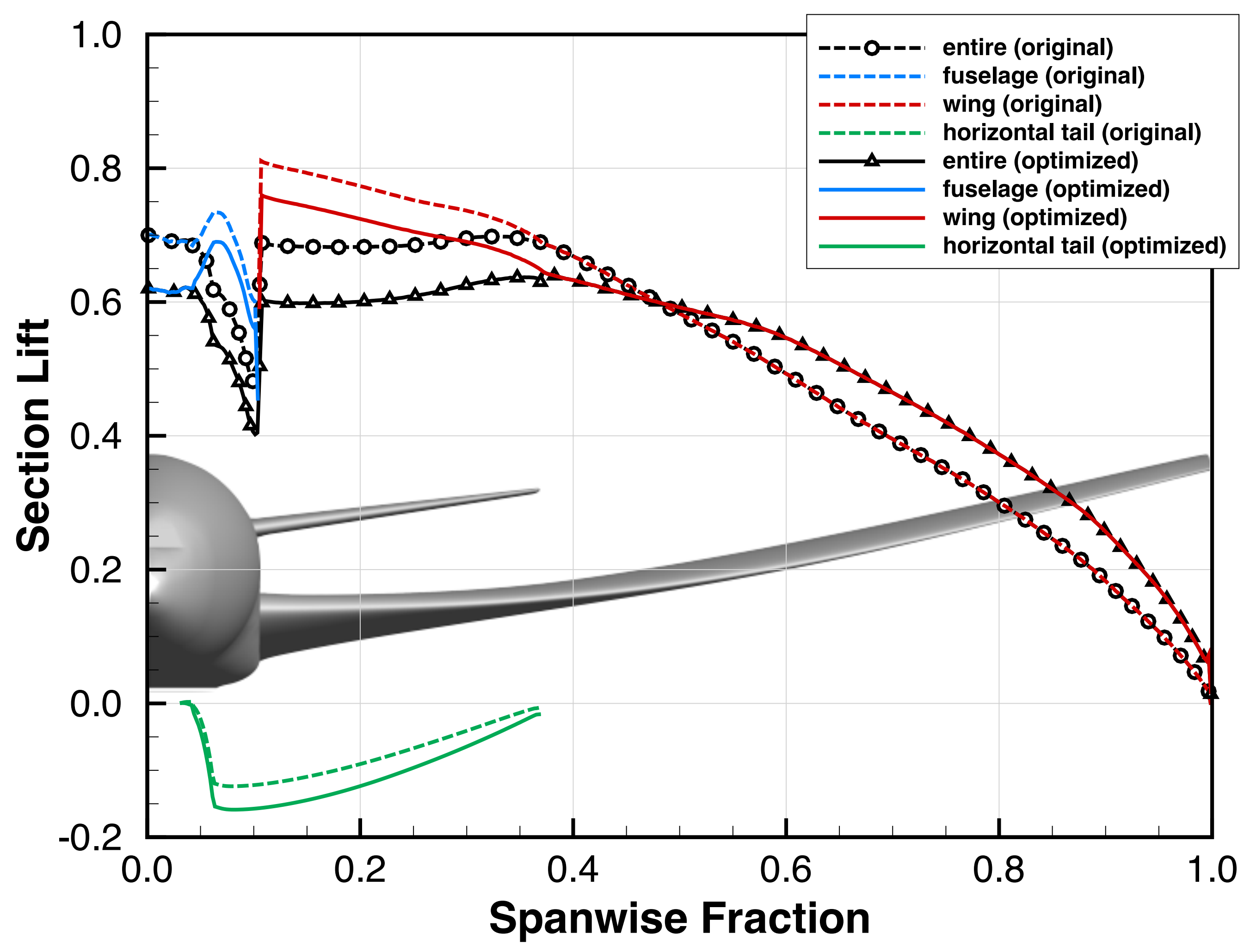




\section{Surface Pressure Distribution}

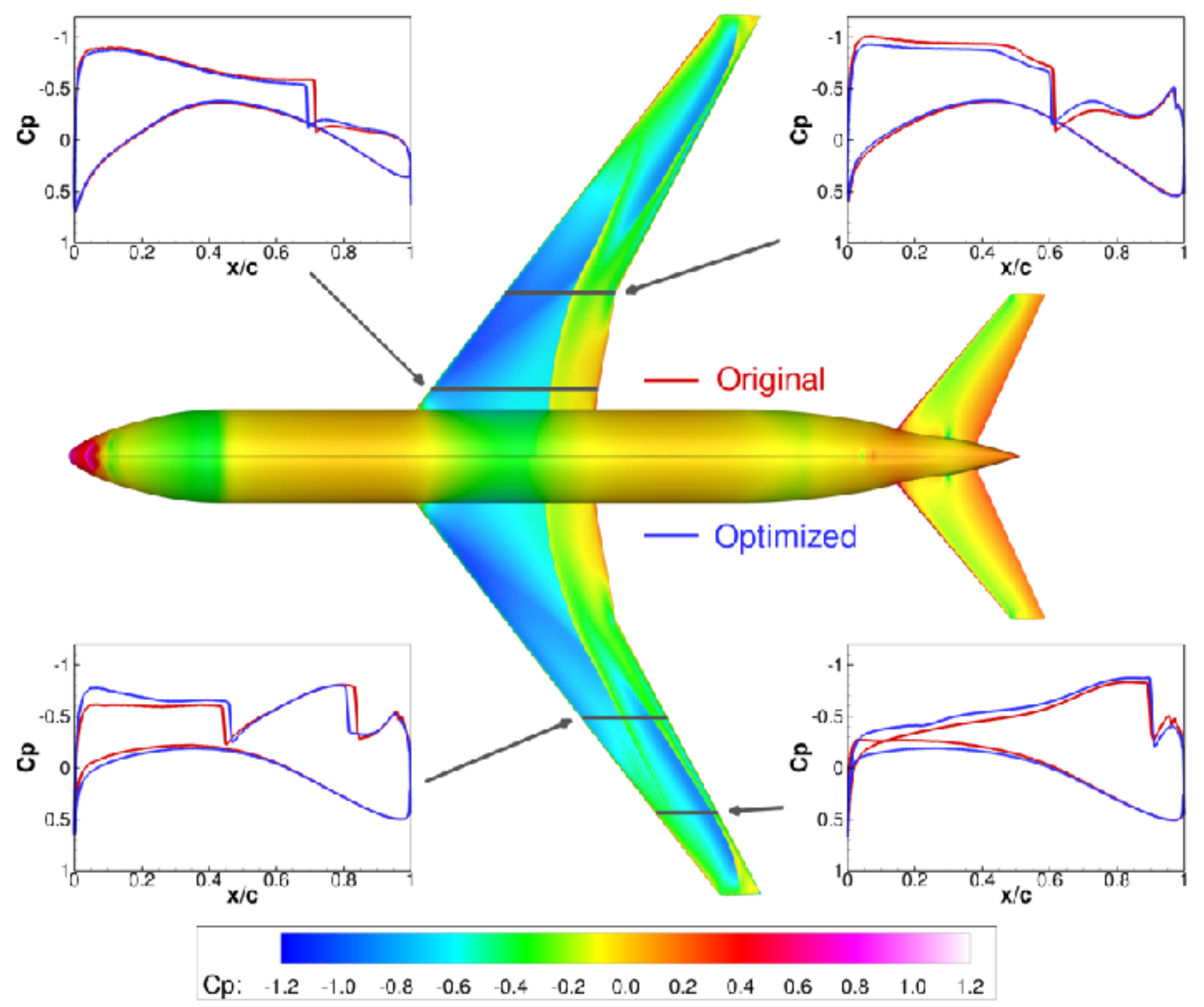




\section{Flap Layout Trade Study}

- Install systems with varying numbers of spanwise flaps $(4,8,12)$ and chordwise segments $(1,2,3)$

- Increase cruise speed

- $M_{\infty}=0.85 \rightarrow M_{\infty}=0.88$

- would save 10 minutes on a 5 hour flight

- Optimize the flap deflections

- minimize drag

- maintain cruise lift

- maintain trim minimize: $\quad C_{D}\left(\alpha, \Delta_{f l a p s}, i_{t}\right) @ M_{\infty}=0.88$

subject to: $\quad C_{L}\left(\alpha, \Delta_{f l a p s}, i_{t}\right)=C_{L}=0.4665$

$C_{M}\left(\alpha, \Delta_{f l a p s}, i_{t}\right)=0$

$\alpha \leq \alpha_{\max }=3^{\circ}$ 


\section{Performance of Optimized 4-Flap Layouts}

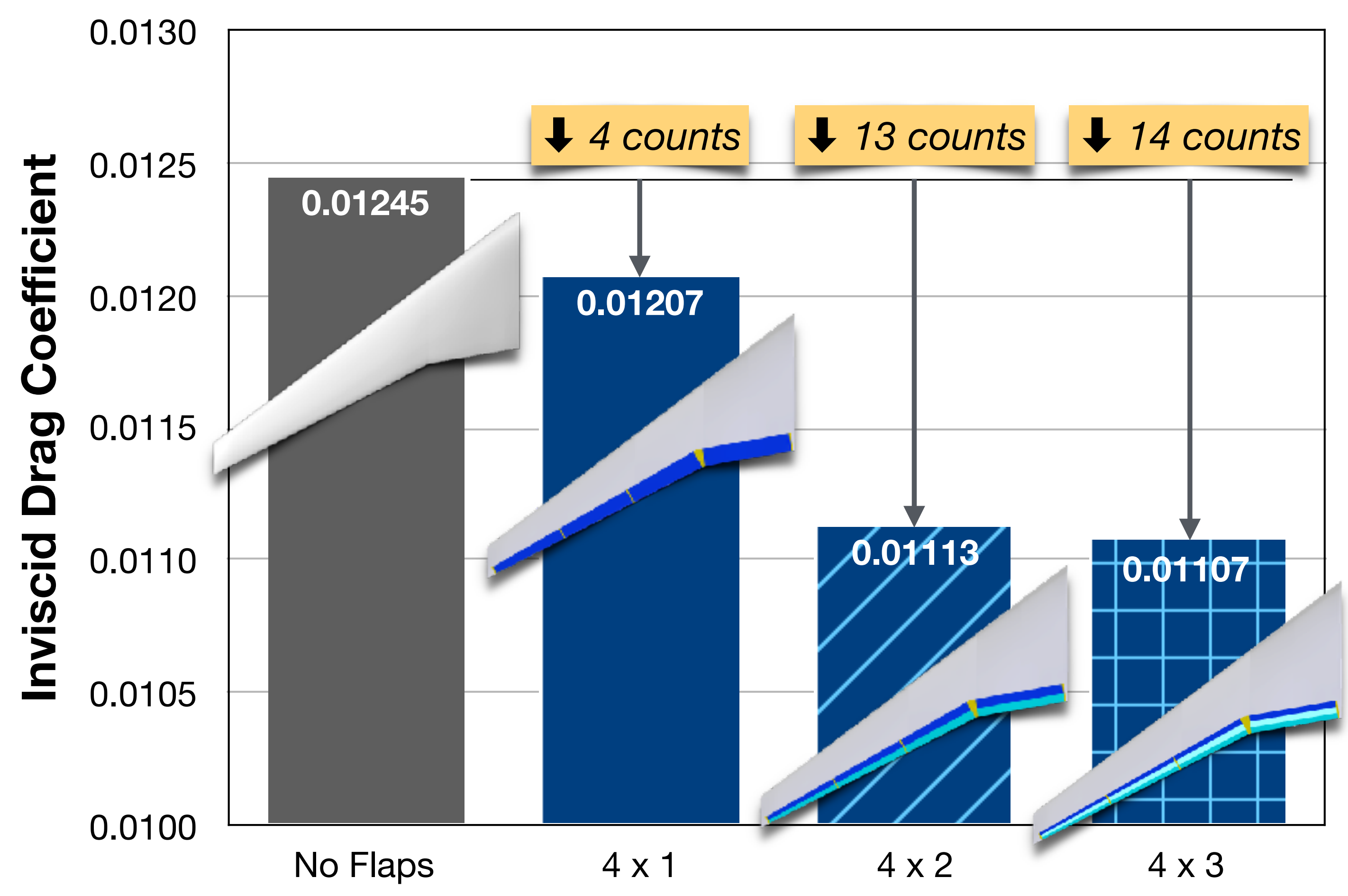

Flap Layout 


\section{Lift Distribution on 4-Flap Systems}

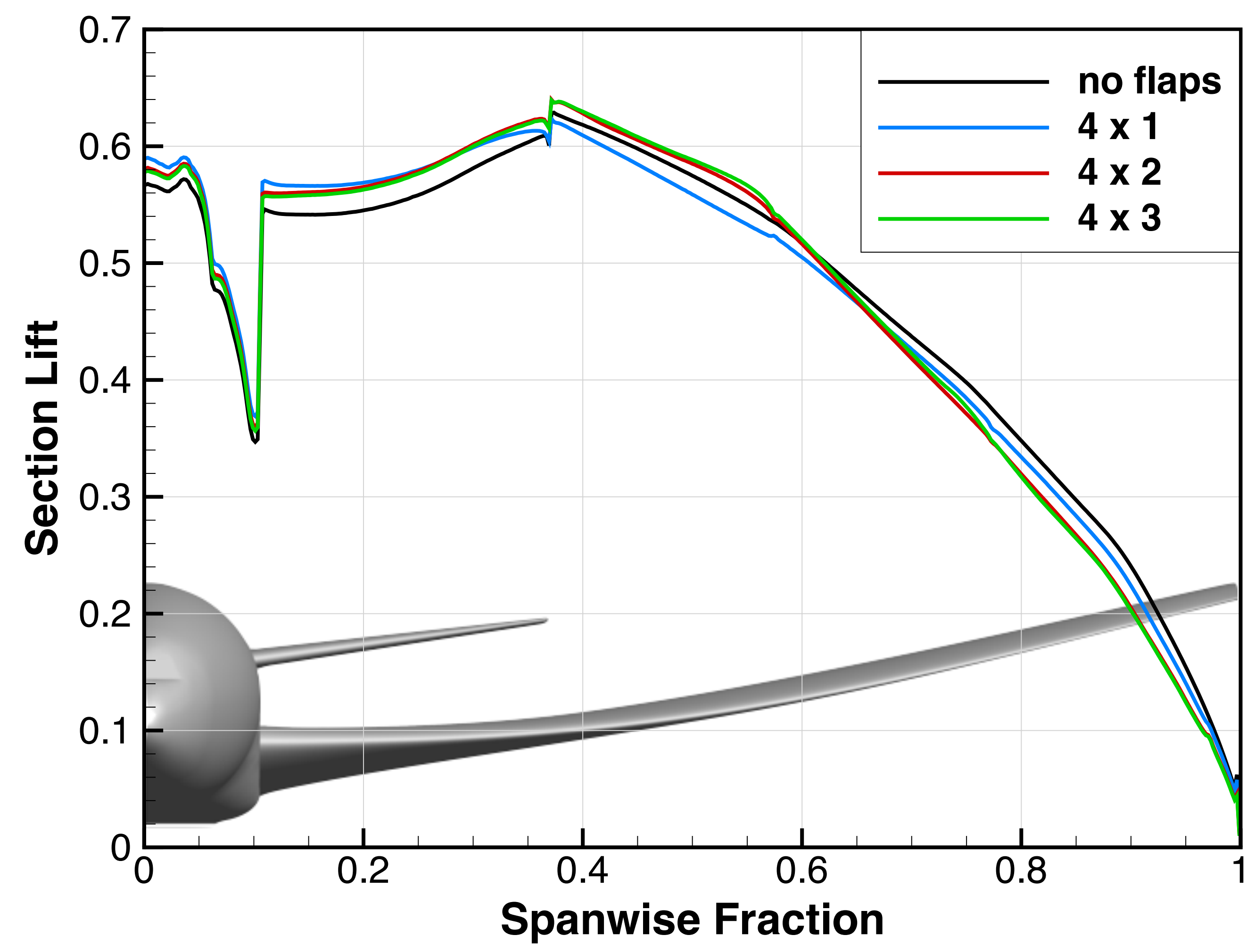




\section{Pressure Distributions on 4-Flap Layouts}
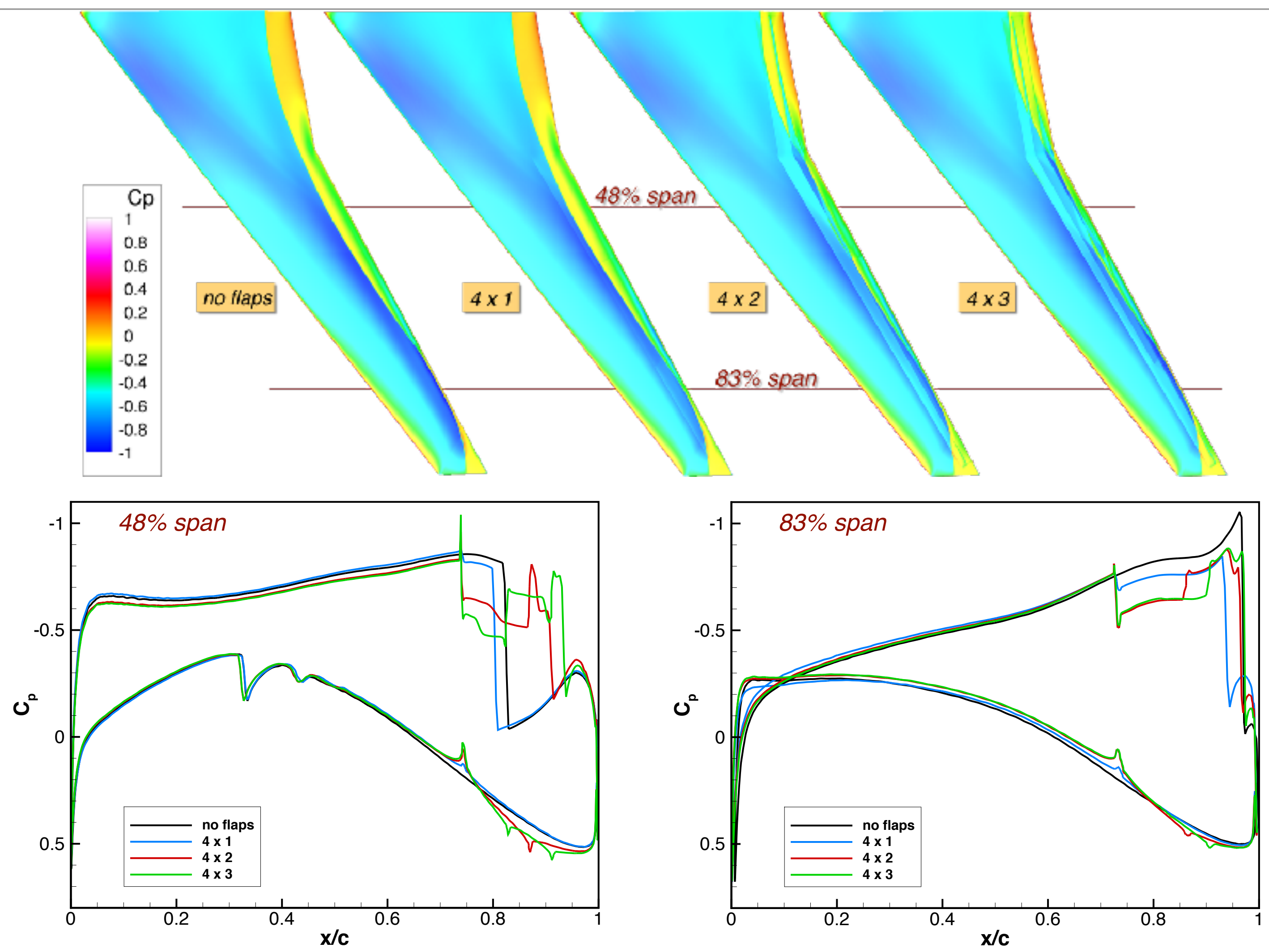


\section{Optimized 4-Flap Geometry}

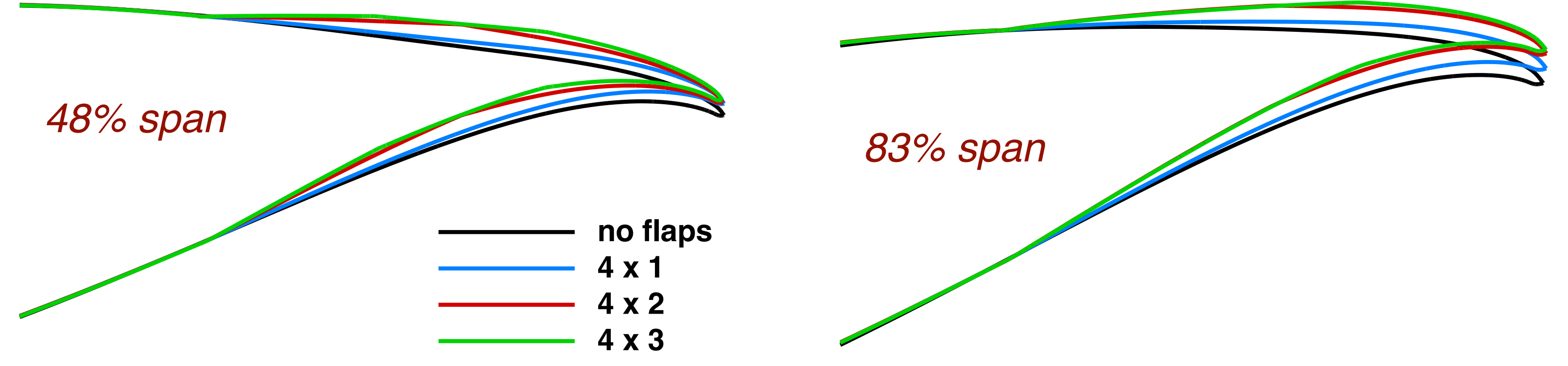

vertical scale is 4 times greater than horizontal for clarity

- 3-segment deflected flap profile very similar to 2-segment

- 1-segment deflected flap somewhere in between undeflected geometry and deflected 2-segment flap

- Deflecting flaps moves reflex backward (consistent with supercritical airfoil theory) 


\section{Performance of All Optimized Flap Layouts}

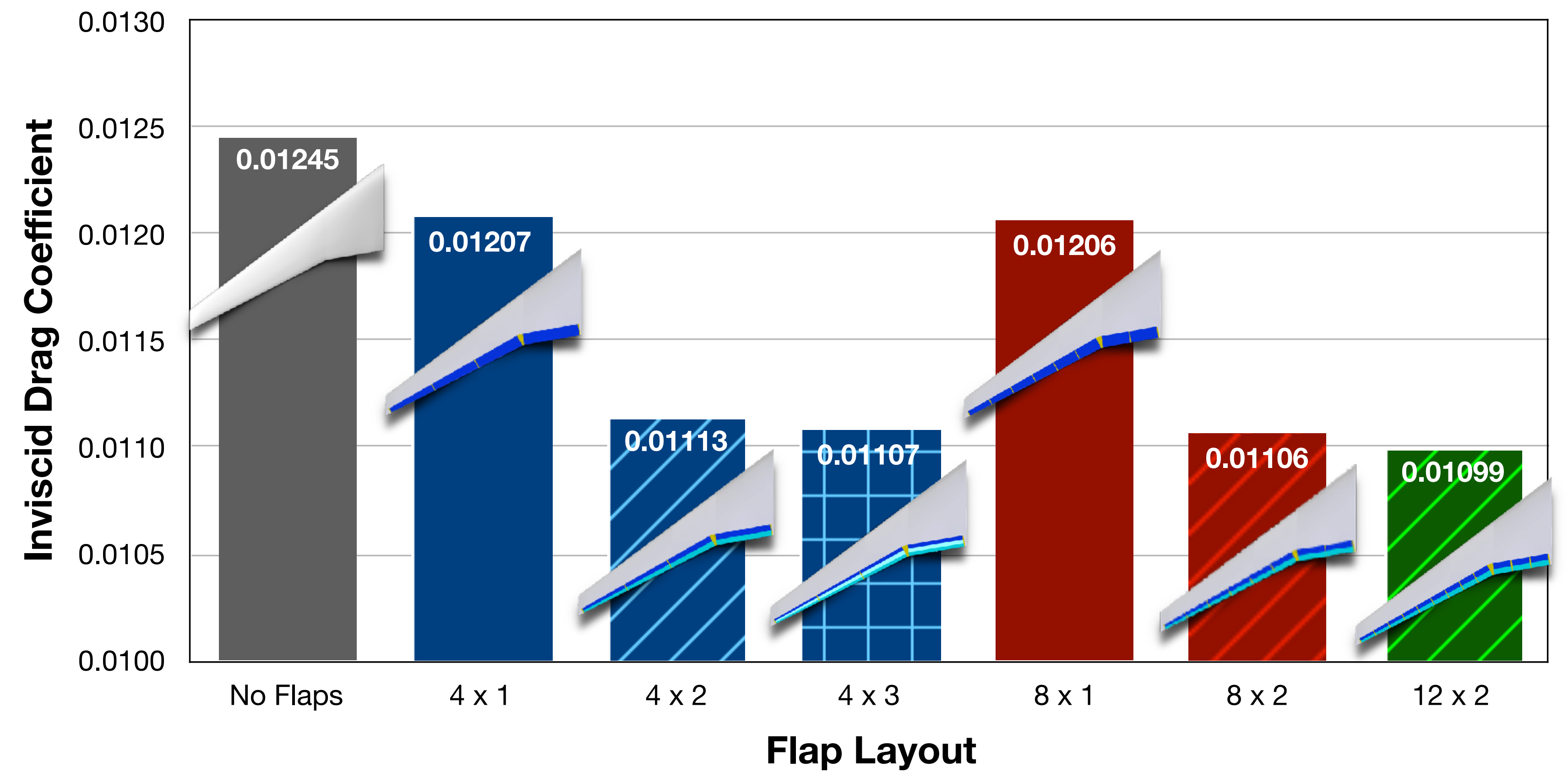




\section{Performance of All Optimized Flap Layouts}

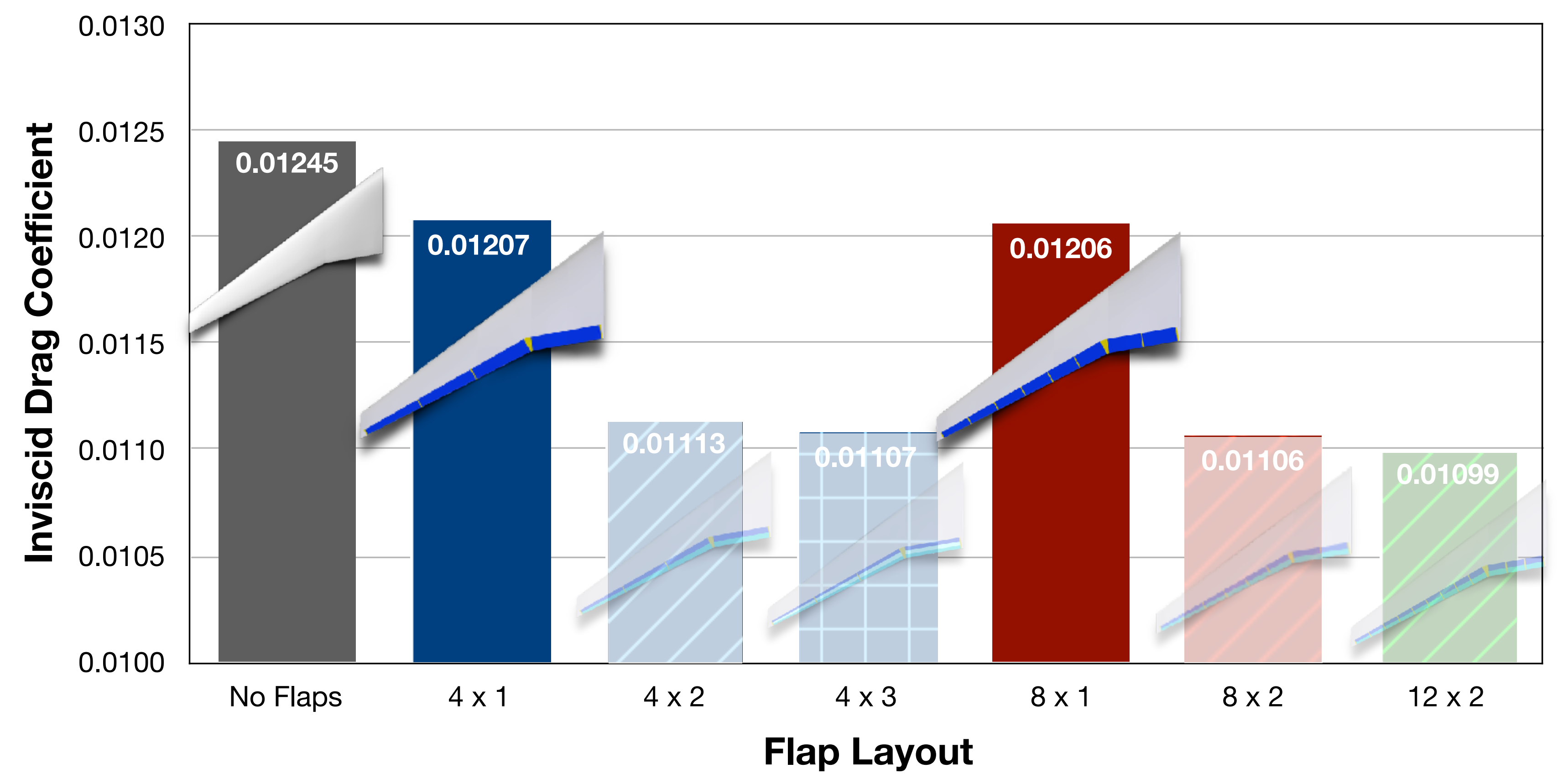




\section{Performance of All Optimized Flap Layouts}

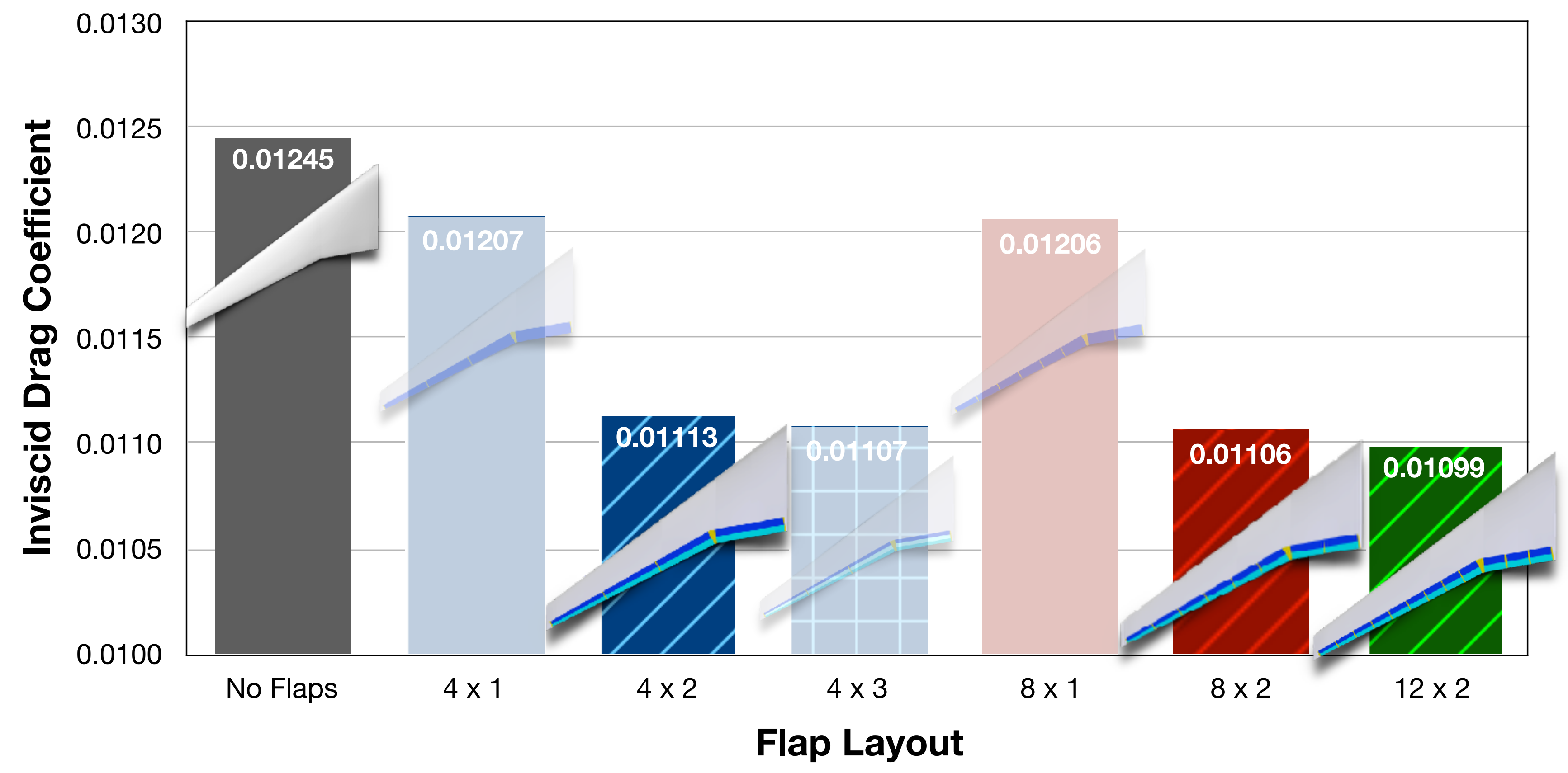


Spanwise Lift Distribution on 2-Segment Systems

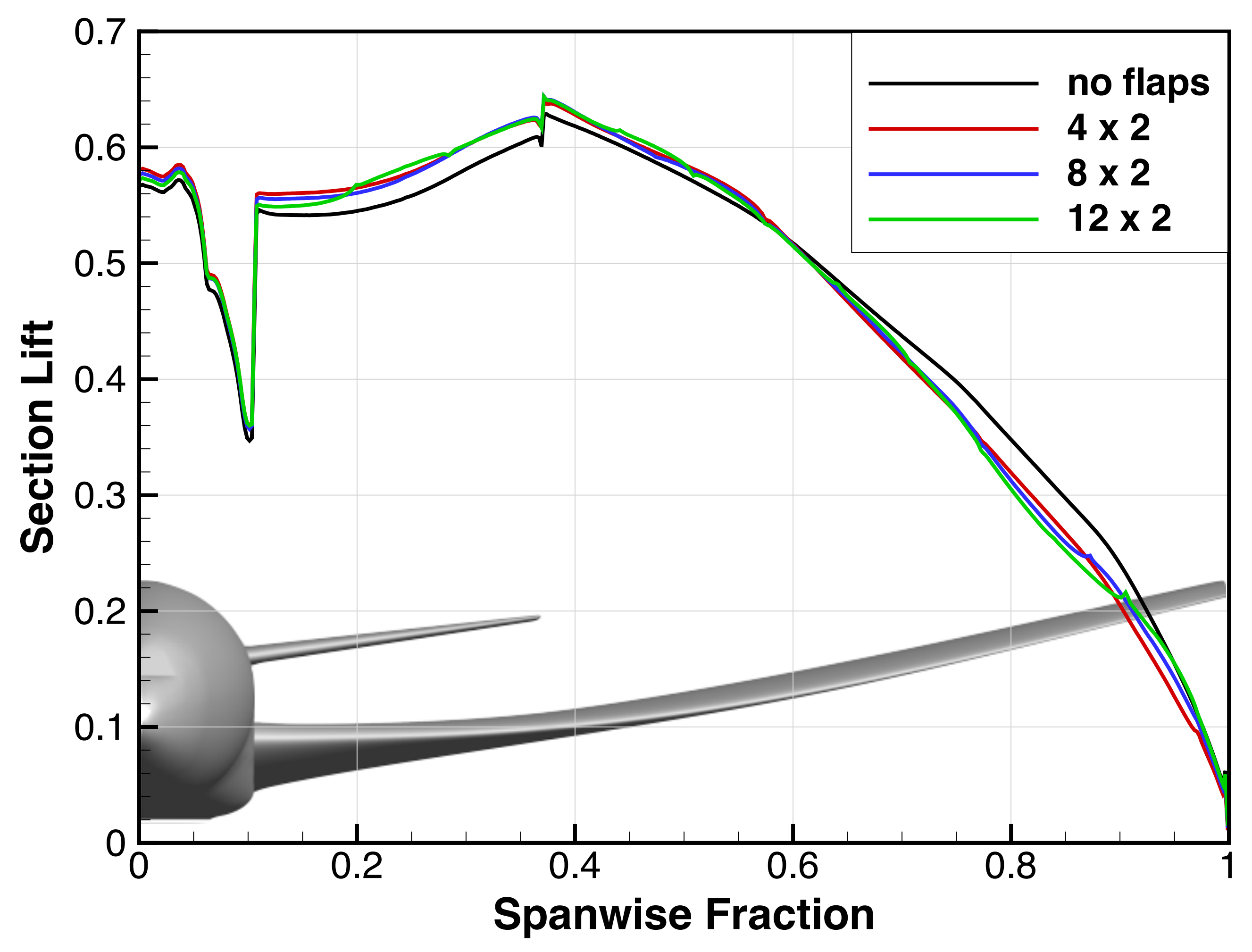




\section{Pressure Distributions on 2-Segment Layouts}
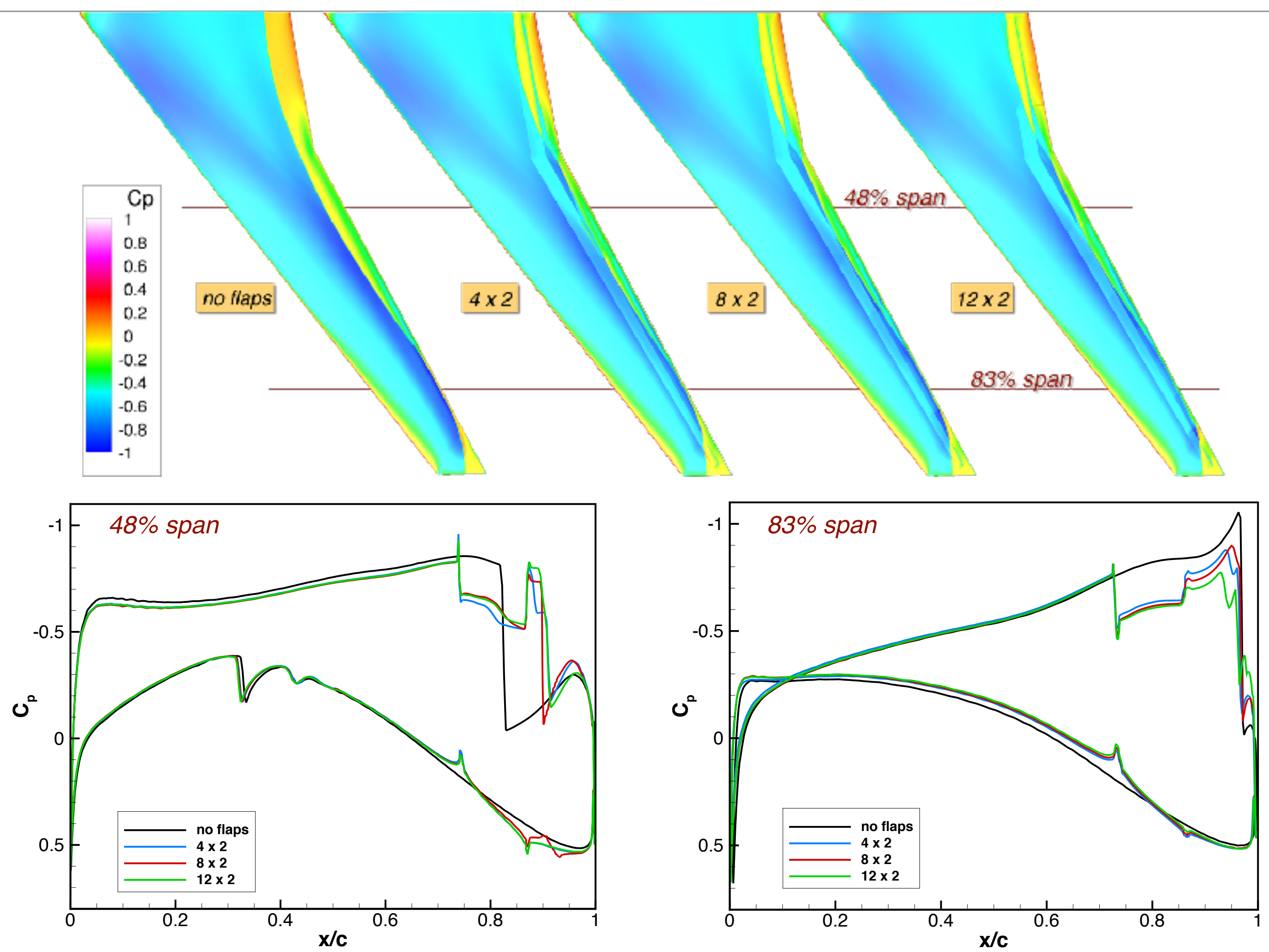


\section{Optimized 4 x 2 Flap Layout Deflections}

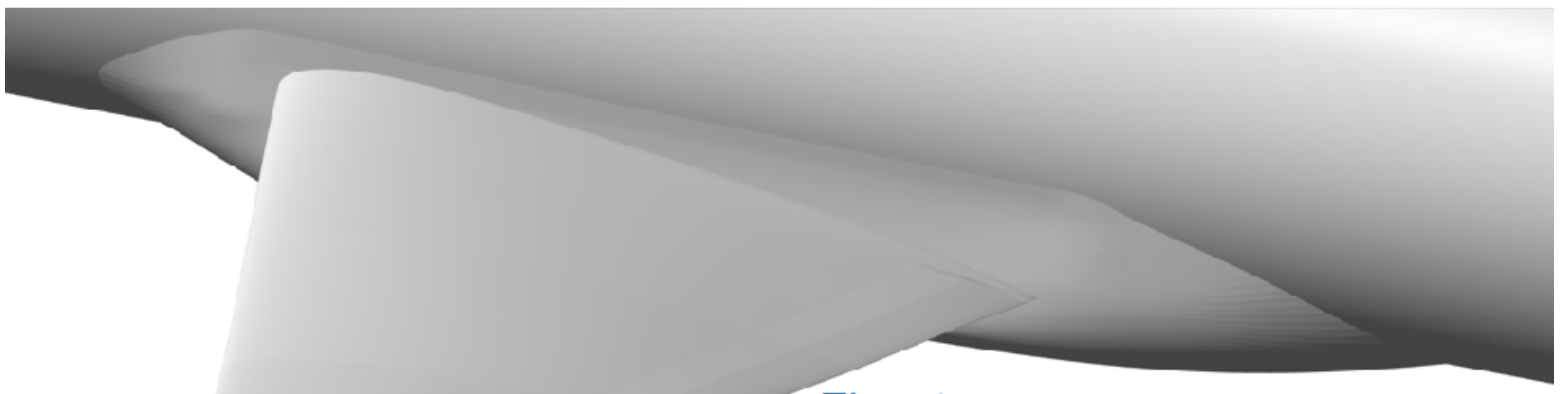

Flap 2

Flap 1

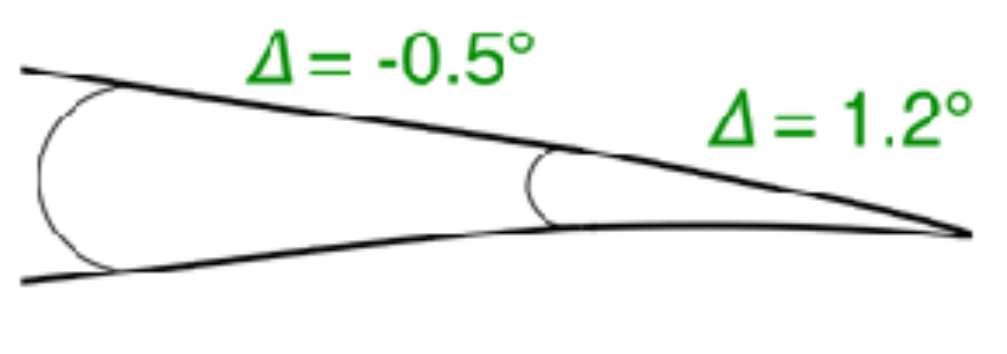

Flap 4
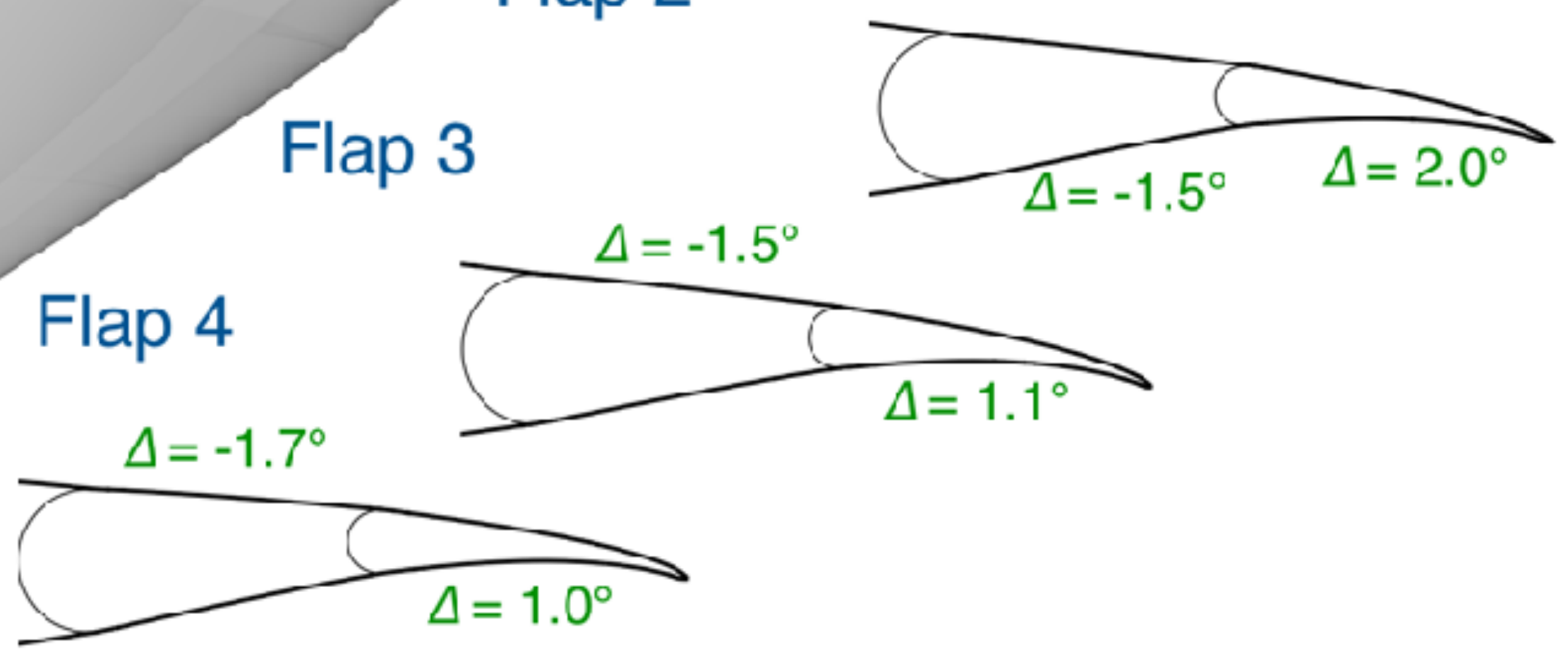


\section{Conclusions and Future Work}

- Flap layout trade study on highly flexible CRM conducted for overspeed off-design case

- 2-segment flaps found to be much more effective than 1-segment flaps, but 3-segment flaps provided only incremental improvement

- 4 spanwise flaps are almost as effective as 12 , suggesting induced drag is either already near optimal or wave drag reduction dominates

- Verification with viscous analysis

- Consider other off-design conditions (e.g. maneuver condition) 


\section{Acknowledgements}

- NASA Advanced Air Transport Technology Project provided partial funding for this work

- NASA Advanced Supercomputing (NAS) Center provided computing resources 